\title{
Role of reactive oily bubble in apatite flotation
}

\author{
Fang Zhou ${ }^{1 *}$, Louxiang $\mathrm{Wang}^{3}$, Zhenghe $\mathrm{Xu}^{3}$, Yaoyang Ruan ${ }^{2}$, Zhenyue \\ Zhang $^{2}$, Ruan Chi ${ }^{1,2}$
}

\begin{abstract}
The effect of fatty acid reactive oily bubble as a carrier in apatite flotation is examined by zeta potential, zeta potential distribution, induction time and micro flotation experiments, and further analyzed by the extended DLVO theory. The surface property of fatty acid reactive oily bubbles is determined by the dissociation degree of fatty acid contained in the oil film covered on reactive oily bubbles. Induction time tests show that the fatty acid reactive oily bubble presents a strong collecting power to apatite particles among $\mathrm{pH} 4.0-11.2$, which is also confirmed by the zeta potential distribution and micro flotation experiments. However, the DLVO theory is fail to predict the coagulation at $\mathrm{pH}$ 9.01. A hydrophobic interaction is considered in the coagulation between reactive oil bubbles and apatite particles. The superiority of fatty acid reactive oily bubble system was confirmed and illustrated by the extended DLVO theory successfully.
\end{abstract}

Keywords: fatty acid, reactive oily bubble, apatite, interaction

\section{Introduction}

Reactive oily bubbles were defined as air bubbles covered with a thin layer of kerosene containing oil-soluble collectors and successfully applied to floating apatite

\footnotetext{
${ }^{*}$ Corresponding author. Tel: +86-18995614810;

E-mail address: $18995614810 @ 163 . c o m$
} 
and dolomite in our previous study [1]. When the reactive oily bubble as a carrier approaches the mineral surface in the flotation process, the collectors at the oil/aqueous interface react selectively with the wanted mineral surface leading to a coagulation. The flotation selectivity can be adjusted by the surface properties of reactive oily bubbles through adding certain type and different concentrations of water insoluble collectors into the oil film $[1,2]$.

The conventional air bubble flotation has a wide range of applications in mineral processing industry, which allows the wanted mineral particles to attach air bubbles to the upper of slurry, while the unwanted mineral particles are left in slurry non-attachable to air bubbles [3]. The flotation is a complicated and heterogeneous separation process, involving the following three steps:

1) Generation of hydrophobicity/hydrophilicity mineral surface, i.e. floatability or non-floatability mineral particles.

2) Contact and interaction of mineral particles to air bubbles and then rise to the upper froth or enter the slurry.

3) Separation of the floatable (wanted) and non-floatable (unwanted) mineral particles [4].

Where, the step two plays an important role in the flotation process. For the reactive oily bubble technology, it has been proven that reactive oily bubbles show a stronger collecting power than air bubbles [1,2,5], although reactive oily bubbles as the mineral particle carrier perform the same role to air bubbles in flotation. To further realize the role of reactive oily bubble in apatite flotation, 100 ppm fatty acid was used as the oil-soluble collector to produce reactive oily bubbles in this study. The zeta potential distribution and the induction time measurements were conducted to evaluate the interaction between apatite particles and bubbles. The extended DLVO theory was applied to analyze the interaction energy between apatite particles and air bubbles/reactive oily bubbles.

\section{Materials and methods}

\subsection{Materials}

Kerosene from Fishier Scientific was applied as the model oil, while fatty acid was used as the apatite collector. Reagent grade $\mathrm{HCl}$ and $\mathrm{NaOH}$ (Fisher Scientific) were used as $\mathrm{pH}$ modifiers. Ultra-high purity $\mathrm{KCl}$ (>99.999\%) from Fisher Scientific was used to prepare $1 \mathrm{mM} \mathrm{KCl}$ solutions as supporting electrolytes. Apatite chunks purchased from Wards Natural Science Establishment (Canada) were hand-crushed 
by a hammer, ground by a Mortar Grinder and then sieved to obtain particles in different size fractions for the following measurements. The apatite was analyzed by the X-Ray Fluorescence micro-probe (EDAX ORBIS XRF) and the X-ray diffraction using a RIG-AKU Rotating anode XRD system (Rigaku RU-200B), respectively. The results were shown in Fig. 1 and Table 1.

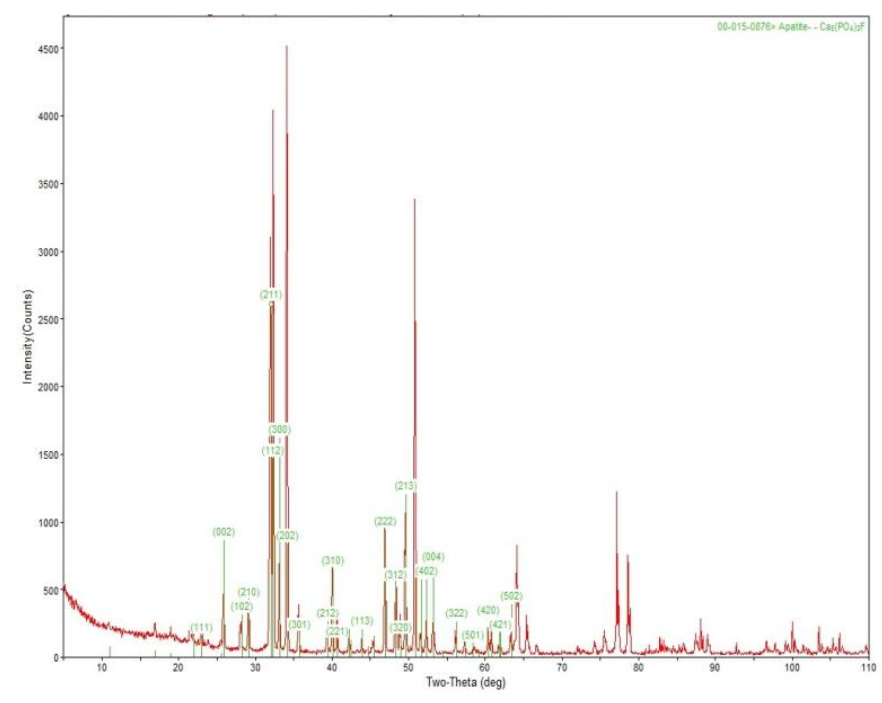

Fig. $1 \mathrm{X}$-ray diffraction of apatite

Table 1 Composition of apatite used in this study (wt\%)

\begin{tabular}{cccccccccc}
\hline Composition & $\mathrm{Na}_{2} \mathrm{O}$ & $\mathrm{MgO}$ & $\mathrm{Al}_{2} \mathrm{O}_{3}$ & $\mathrm{SiO}_{2}$ & $\mathrm{P}_{2} \mathrm{O}_{5}$ & $\mathrm{SO}_{3}$ & $\mathrm{CaO}$ & $\mathrm{CeO}_{2}$ & $\mathrm{Fe}_{2} \mathrm{O}_{3}$ \\
\hline Apatite & 2.19 & - & 0.21 & 1.36 & 46.06 & 1.11 & 48.18 & 0.77 & 0.12 \\
\hline
\end{tabular}

As shown in Fig.1 and Table 1, the XRD peaks of the tested mineral sample matched closely with that of the standard apatite sample, and its main composition is $\mathrm{P}_{2} \mathrm{O}_{5}$ (46.06 wt\%) and $\mathrm{CaO}$ (48.18 wt\%). Both of the XRD and XRF results confirm the apatite sample.

\subsection{Zeta potential and zeta potential distribution measurement}

Zeta potential and zeta potential distribution of reactive oily bubbles and apatite particles were measured using a Zetaphoremeter III (SEPHY/CAD) at ambient temperature of $22 \pm 0.5{ }^{\circ} \mathrm{C}$.. The concrete equipment description and the details on the experimental procedures can be found elsewhere [1,2,5]. A $1 \mathrm{mM} \mathrm{KCl}$ solution was used as the supporting electrolyte for this experiment. For each experimental condition, the zeta potential distribution measurement was repeated at least three times for the same sample solution and nine sets for a tested sample. The average values as well as the standard deviations as error bars were reported. 


\subsection{Interaction measurement}

Interactions between reactive oily bubbles and apatite particles were evaluated by the induction time and zeta potential distribution measurements. For zeta potential distribution measurement, the ZetaphoremeterIII (SEPHY/CAD) can trace 50-100 particles simultaneously and provides a zeta potential distribution histogram, which is a good method to investigate the interaction, i.e., coagulation phenomena, between reactive oily bubbles and apatite particles. The zeta potential distribution of reactive oily bubbles is distinct from apatite particles at different $\mathrm{pHs}$. When the reactive oily bubbles and apatite particles are mixed, the two coagulates or not can be assessed by the presence of only one zeta potential distribution peak or two distinct zeta potential distribution peaks detected. The more detailed description on this technique can be found in literatures $[6,7]$.

The visual interaction between the reactive oily bubble and the apatite particle was directly investigated by the induction time measurement. For each induction time measurement, a single reactive oily bubble was moved towards to an apatite particle bed on a silicon cell with conditioned solutions. For a certain contact time, if the reactive oily bubble attaches to apatite particles, a coagulation can be observed by the macro lens connected to the CCD camera. If there is no coagulation, the reactive oily bubble without attached apatite particles will be detected. The method and detail procedure description was given in literatures $[1,5,8,9]$.

\subsection{Micro flotation}

In each micro flotation experiment, $5 \mathrm{~g}$ of apatite particles (particle diameter 45-75 $\mu \mathrm{m})$ was dispersed in de-ionized water in a beaker to make the needed mineral suspension for flotation. $900 \mathrm{~mL}$ of the conditioned mineral suspension was then placed in a modified micro-flotation cell for $5 \mathrm{~min}$ flotation. After that, the concentrates and the tailings were collected, filtered, dried and weighed. The percentage of the concentrates was calculated as the flotation recovery being plotted as a function of the solution $\mathrm{pH}$. The schematic illustration of micro-flotation and a more detailed description of this technique were given in our previous papers $[1,5]$.

\subsection{Estimation of interaction energy}

In the fine particle flotation system, the interaction energy between mineral particles and bubbles can be explained by the classic DLVO theory $[10,11,12]$. However, non-DLVO force such as hydrophobic forces cannot be ignored with the development in direct force measurement, which are included into the classic DLVO theory, named 
the extended DLVO theory $[13,14,15]$. The interaction energies between reactive oily bubbles and apatite particles in this study is estimated by the extended DLVO theory as follows:

$E_{t}=E_{e}+E_{a}+E_{h}$

Where $E_{e}$ is the electrostatic interaction energy, $E_{a}$ is the London-van der Waals energy and $E_{h}$ is the hydrophobic energy.

\subsubsection{Electrostatic interaction energy}

The electrostatic interaction energy $E_{e}$ can be calculated by the following equation.

$E_{e}=\frac{\varepsilon_{0} \varepsilon_{r} R_{1} R_{2}\left(\zeta_{1}^{2}+\zeta_{2}^{2}\right)}{4\left(R_{1}+R_{2}\right)}\left[2 \frac{\zeta_{1} \zeta_{2}}{\zeta_{1}^{2}+\zeta_{2}^{2}} \ln \frac{1+\exp (-\kappa H)}{1-\exp (-\kappa H)}+\ln (1-\exp (-2 \kappa H)]\right.$

in which $\varepsilon_{0}$ is the dielectric constant under vacuum, $8.85 \times 10^{-12}\left(\mathrm{C}^{2} \mathrm{~J}^{-1} \mathrm{~m}^{-1}\right)$, $\varepsilon_{\mathrm{r}}$ is the relative dielectric constant of water, $\zeta_{1}$ and $\zeta_{2}$ are the tested zeta potentials of reactive oily bubble and mineral particle. $K$ is the Debye reciprocal length which can be determined by the solution electrolyte concentration represents the thickness of the electrical double layer. From the classic Debye-Hucel theory, $1 / K$ is calculated as 10 $\mathrm{nm}$ for $1 \mathrm{mM} \mathrm{KCl}$ solutions (1:1 electrolyte) at $25{ }^{\circ} \mathrm{C}[14,16]$.

\subsubsection{London-van der Waals energy}

For reactive oily bubble flotation system, the reactive oily bubble and the apatite particle were regarded as sphere 1 and sphere 2 , and their radius are $R_{1}$ and $R_{2}$, respectively. The London-van der Waals energy among them is thus calculated as following:

$$
E_{a}=-\frac{R_{1} R_{2} A_{132}}{6\left(R_{1}+R_{2}\right) H}
$$

where, $A_{132}$ is the complex Hamaker constant for the reactive oily bubble flotation system, which is given as follows $[15,17]$ :

$$
A_{132}=\left(\sqrt{A_{11}}-\sqrt{A_{33}}\right)\left(\sqrt{A_{22}}-\sqrt{A_{33}}\right)
$$

where $A_{11}, A_{22}, A_{33}$ refer to the Hamaker constants of the sphere 1 (reactive oily bubble), sphere 2 (apaite particle) and medium 3 (water) respectively. Eq. (3) indicates that if $A_{132}$ is negative, the $E_{\mathrm{a}}$ is positive and the electrostatic interaction force is attractive; otherwise the electrostatic interaction force is repulsive. 


\subsubsection{Hydrophobic energy}

Mineral flotation is a complex liquid-solid-bubble process, which is very difficult to be explained by a single flotation simulation. Many flotation simulations proposed by researchers are the empirical model [18,19,20]. Dobby and Finch [21] developed hydrodynamic collision models. However, an important parameter in mineral flotation process, the surface chemistry parameter is neglected. With the development of surface forces measurement (SFA and AFM), many ignored elements in the past are considered and introduced into the DLVO theory. Researchers found that the hydrophobic energy plays an important role in the collision, coagulation and dispersion of fine particles in liquid phase [22,23,24]. Israelachvili and Pashley [25] showed a non-DLVO force between mica surfaces immersed in cetyltrimethylammonium bromide solution by direct force measurements, which was much stronger than the London-van der Waals force. This confirmed hydrophobic force is given as follows [26,27,28]:

$$
\frac{F}{R}=C_{1} \exp \left(\frac{H}{D_{1}}\right)+C_{2} \exp \left(\frac{H}{D_{2}}\right)
$$

where $C_{1}$ and $C_{2}$ are the pre-exponential parameters, $D_{1}$ and $D_{2}$ are decay lengths. Eq. (5) is applied generally with $D_{1}$ in the range of 1-2 nm and $D_{2}$ in the range of 10-32 $\mathrm{nm}$ [29]. The hydrophobic forces measured can be also fitted by a power law [30].

$$
\frac{F}{R}=-\frac{K}{6 H^{2}}
$$

where $K$ is the hydrophobic force parameter. Eq. (6) has only one parameter $(K)$ and is of the same forms as Eq. (3). Thus, the London-van der Waals force can be compared directly with the hydrophobic force by the values of $K$ and $A$ (Hamaker constant).

Although the origin of hydrophobic force is not completely understood, it is still confirmed in the experiments, which make the bubble-particle interaction possible by the extended DLVO theory [31,32,33]. From Eq. (5) and Eq. (6), the hydrophobic energy is given as follows:

$$
E_{h}=-\frac{1}{2} \int_{\infty}^{H} F_{h} d H
$$

where $H$ is interaction distance, $F_{\mathrm{h}}$ is the hydrophobic force. When Eq. (6) is substituted into Eq. (7), the hydrophobic energy is given as follows: 
$E_{h}=-\frac{R_{1} R_{2}}{6\left(R_{1}+R_{2}\right)} \frac{K_{132}}{H}$

in which the constant $K_{132}$ can be approximate expressed as the following [34]:

$$
K_{132}=\sqrt{K_{131} K_{232}}
$$

where $K$ is the hydrophobic force parameter for bubble (1)-particle (2) interaction in medium (3). Aksoy [35] showed that the particle-particle interaction $\left(K_{131}\right)$ of bubble is less than $10^{-17} \mathrm{~J}$ in the presence of surfactant. For the most of hydrophobic surface, as well as the contact angle larger than $90^{\circ}$, the particle-particle interaction $\left(K_{131}\right)$ is larger than those for air bubbles interaction $\left(K_{232}\right)$.

\section{Results and discussion}

\subsection{Zeta potentials}

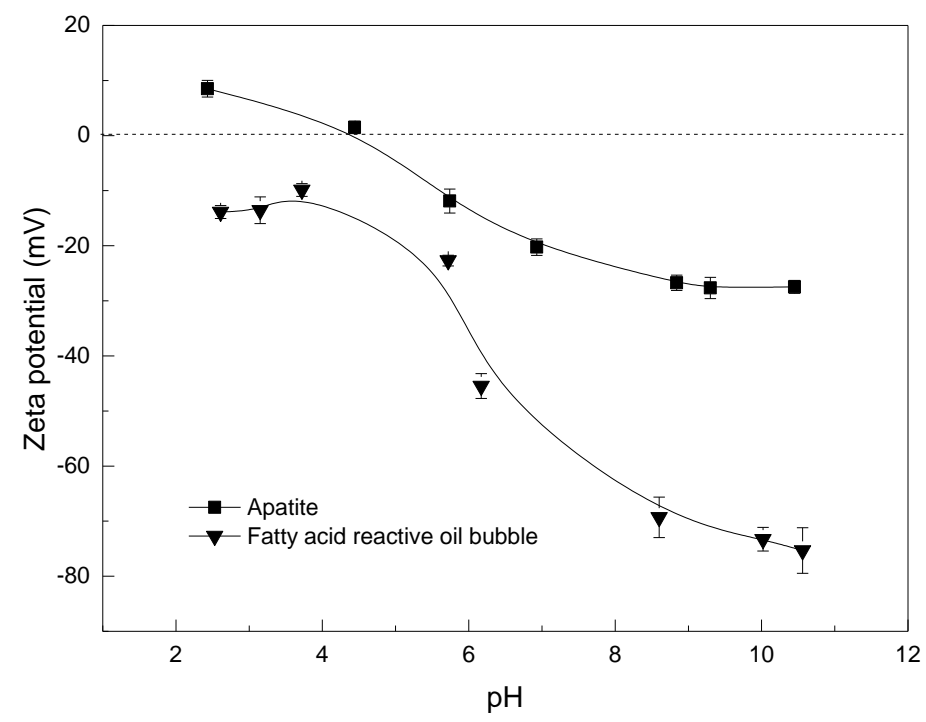

Fig.2 Zeta potentials as a function of $\mathrm{pH}$

From Fig.2, the zeta potentials of reactive oily bubbles are negative over the tested $\mathrm{pH}$ range from 2 to 11 . For apatite particles, an isoelectric point (IEP) was investigated at about $\mathrm{pH} 4.2$, suggesting that the surface charge of apatite is positive over a range of $\mathrm{pH} 2-4.2$, and negative over a range of $\mathrm{pH}$ 4.2-10.5. A coagulation is expected theoretically by the opposite surface charge between reactive oily bubbles and apatite particles around $\mathrm{pH} 2-4.2$ on account of electrostatic interaction, and no coagulation over a range of $\mathrm{pH}$ 4.2-10.5 correspondingly. However, electrostatic interaction energy is not the only factor to determine the coagulation of reactive oily bubbles and 
apatite particles. The coagulation of reactive oily bubbles and apatite particles is also affected by the London-van der Waals energy and hydrophobic energy.

\subsection{Surface property of reactive oily bubble}

Fatty acid used as oil-soluble collectors in this study is a hydrolysable weak acid surfactant, which hydrolyzes to fatty acid anion in aqueous phase. The hydrolysis equation in aqueous phase is given as:

$$
\mathrm{RCOOH} \leftrightarrow \mathrm{RCOO}+\mathrm{H}^{+}
$$

The acid dissociation constant of fatty acid, $p K a$, is about $4.95[36,37]$, which can be given as:

$$
K_{a}=\frac{\left[\mathrm{RCOO}^{-}\right]\left[\mathrm{H}^{+}\right]}{\mathrm{RCOOH}}=10^{-4.95}
$$

The dissociation degree of fatty acid, $\alpha$, is given as the following:

$$
\alpha=\frac{\text { Number of dissociated molecule }}{\text { Number of molecule }}=\frac{\left[\mathrm{RCOO}^{-}\right]}{\left[\mathrm{RCOO}^{-}\right]+[\mathrm{RCOOH}]}=\frac{10^{-4.95}}{\left[\mathrm{H}^{+}\right]+10^{-4.95}}
$$

Assume that the ion concentration distribution on the oil-water boundary surface is in line with the Boltzmann distribution law.

$$
C=C_{0} \exp \left(-E_{P} / K T\right)
$$

where $C$ is the ion concentration when $E_{\mathrm{P}}=E_{\mathrm{P}}, C_{0}$ is the ion concentration when $E_{\mathrm{P}}=0$. $E_{\mathrm{P}}$ is the potential energy in the external force field, $K$ is the Boltzmann constant. In the electric potential field, $E_{\mathrm{P}}$ is:

$$
E_{P}=Z F \varphi_{0}
$$

in which $Z$ is the number of valence electrons, $F$ is the charge electron, $\varphi_{0}$ is surface charge, substituted for zeta potential, $\zeta(\mathrm{mV})$. Substituting Eq.(14) into Eq.(13), the ion Boltzmann distribution of Eq. (15) is obtained as follows:

$$
C=C_{0} \exp \left(-Z F \varphi_{0} / K T\right)
$$

The relationship of the $\mathrm{H}^{+}$concentration on oil-water interface, $C_{o i l}^{H^{+}}$, and the $\mathrm{H}^{+}$ concentration in the aqueous phase, $C^{H^{+}}$, is given as follows:

$$
C_{\text {oil }}^{H^{+}}=C^{H^{+}} \exp (-Z F \zeta / K T)
$$

After logarithm, Eq.(16) is transformed into Eq.(17): 


$$
p H_{o i l}=p H+\lg (\exp (-Z F \zeta / K T))
$$

That is,

$$
p H_{o i l}=p H-Z F \zeta / K T \ln 10=p H-Z F \zeta / 2.3 K T=p H-17.3 \zeta
$$

From Eq.(12), the relationship of $\mathrm{pH}$ and the dissociation degree of fatty acid is given as follows:

$$
\alpha=\frac{10^{-4.95}}{10^{-p H}+10^{-4.95}}
$$

According to Eq.(12) and Eq.(18), the relationship of $p H_{o i l}$ and the dissociation degree of fatty acid is given as the following:

$$
\alpha=\frac{10^{-4.95}}{10^{-p H_{\text {oil }}-17.3 \zeta}+10^{-4.95}}
$$

To investigate the relationship between the zeta potential of reactive oily bubbles and the dissociation degree of fatty acid, Eq. (19) and Eq. (20) were plotted and compared to the zeta potential of reactive oily bubbles in Fig.2 as a function of $\mathrm{pH}$ shown in Fig.3.

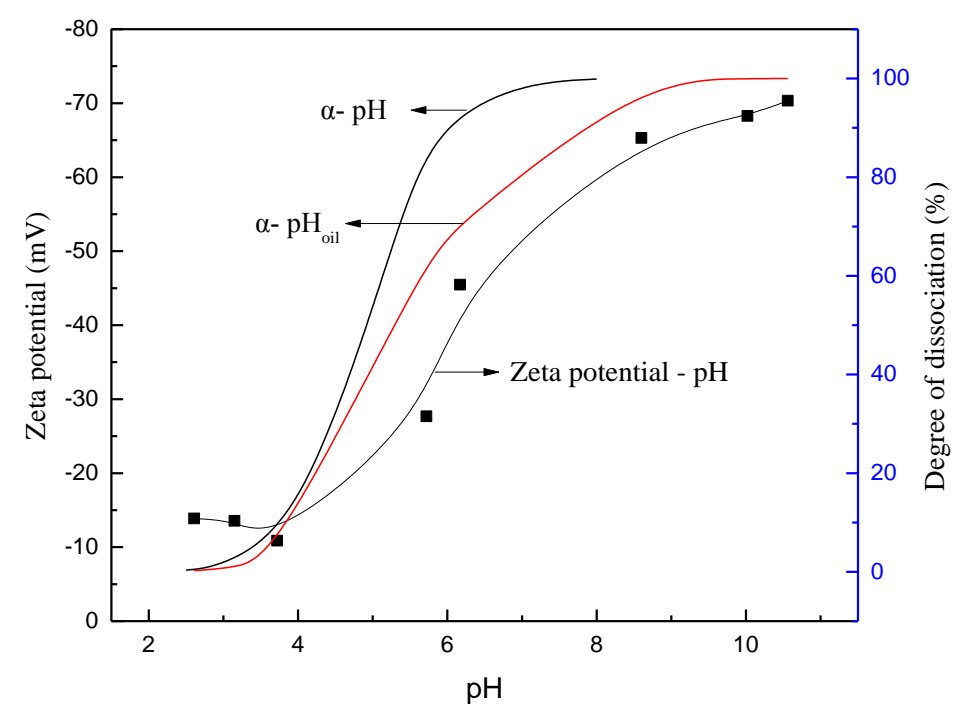

Fig.3 Comparison of zeta potential of reactive oily bubble on dissociation degree as a function of $\mathrm{pH}$

Figure 3 shows that both the dissociation degree-pH line and the dissociation degree- $\mathrm{pH}_{\mathrm{oil}}$ line are with the same variation tendency to the $\zeta-\mathrm{pH}$ line, suggesting the adsorption of surfactant (fatty acid) on the kerosene-water interface when it is added into kerosene to form the reactive oily bubble. The surface property of kerosene-water interface of reactive oily bubble is affected by the surfactant property, i.e. fatty acid 
property, which further indicates the surface property of reactive oily bubble is determined by the added surfactant itself and the solution $\mathrm{pH}$. It is thus concluded that what kind and how much the surfactant is in the oil film of reactive oily bubble plays an important role in the surface property control of reactive oily bubbles.

\subsection{Interaction measurements}

\subsection{1 induction time}

Induction time was measured to investigate directly the interaction between reactive oily bubbles and apatite particles. The results are shown in Fig 4.
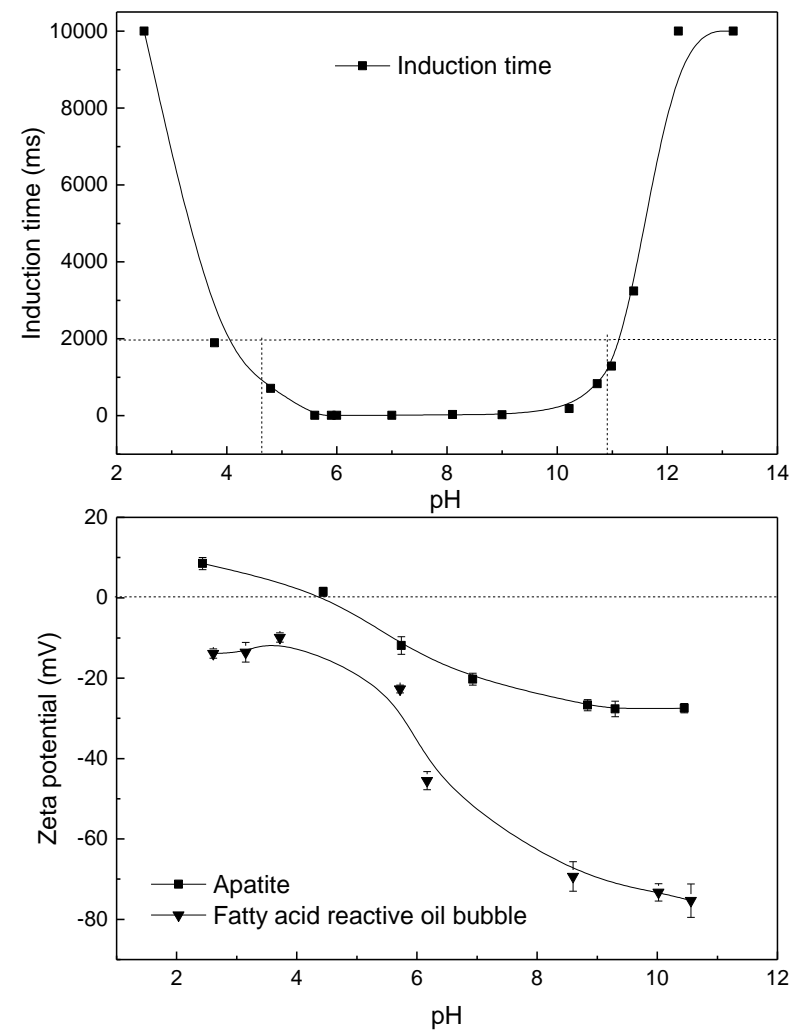

Fig.4 Induction time and Zeta potential of fatty acid reactive oily bubble and apatite as a function of $\mathrm{pH}$

From Fig.4, the induction time of fatty acid reactive oily bubble attached to apatite particles is lower than $2000 \mathrm{~ms}$ around the $\mathrm{pH}$ range of 4-11, suggesting a strong collecting power of the fatty acid reactive oily bubble to the apatite particles in this $\mathrm{pH}$ range [1]. The fatty acid reactive oily bubble shows electronegative in the whole tested $\mathrm{pH}$ range. For the apatite particle, the iep is about 4.2, suggesting that the surface potential of apatite particles is positive when $\mathrm{pH}<4.2$, and negative when $\mathrm{pH}>4$.2. Therefore, an electrostatic attraction force between fatty acid reactive oily 
bubbles and apatite particles at the $\mathrm{pH}<4.2$ and an electrostatic repulsive force at $\mathrm{pH}>4.2$ is expected based on the electrostatic interaction theory. If the electrostatic force is the only one considered in the interaction between fatty acid reactive oily bubbles and apatite particles, a coagulation at $\mathrm{pH}<4.2$ and un-coagulation at $\mathrm{pH}>4.2$ should be observed. However, the induction time results show that the fatty acid reactive oily bubble cannot collect the apatite particles at the strong acidic condition ( $\mathrm{pH} 3$ ). And the fatty acid oily reactive oily bubble collects the apatite particles from $\mathrm{pH} 4.0$ to 11.2, which is opposite to the results deduced from the electrostatic interaction theory. All of the above results confirm that the electrostatic interaction is not the unique factor to determine the coagulation between reactive oily bubbles and apatite particles, and is insufficient to illustrate the complicated interfacial interaction between the reactive oily bubbles and the apatite particles.

\subsubsection{Zeta potential distribution}

To further confirm the induction time results, the zeta potential distribution of the mixture of fatty acid reactive oily bubbles and apatite particles at $\mathrm{pH} 3.12$ and $\mathrm{pH}$ 9.01 were measured and shown in Fig. 5 and Fig.6.

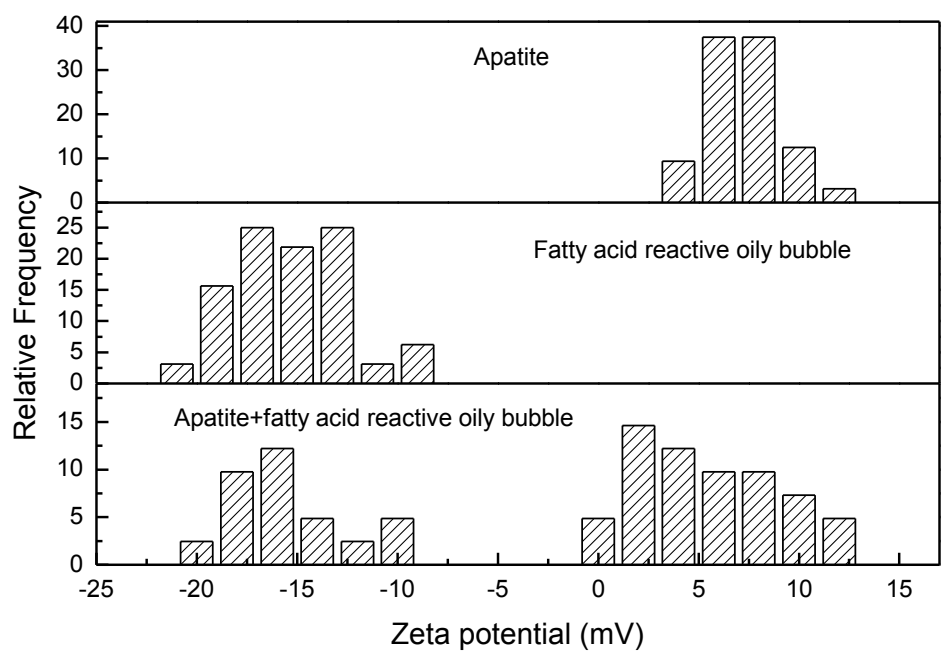

Fig.5 Zeta potential distribution of individual apatite and reactive oily bubble and their mixture at $\mathrm{pH} 3.12$

Figure 5 shows that in the solution of $\mathrm{pH} 3.12$, the zeta potential distribution peak of the fatty acid reactive oily bubbles locates at around $-15 \mathrm{mV}$ and the peak of apatite particles locates at around $8 \mathrm{mV}$. An electrostatic attractive force from the opposite charge and thus a coagulation between the fatty acid reactive oily bubbles and the apatite particles should be investigated from the electrostatic interaction theory. 
However, the tested results in Fig.5 show that the mixture has two distinct zeta potential distributions, and their locations are similar to that of the single bubbles and particles, suggesting that the fatty acid reactive oily bubbles is uncoagulated to the apatite particles at $\mathrm{pH} 3.12$. A stronger repulsive force than the electrostatic attractive force between the fatty acid reactive oily bubbles and the apatite particles may result in this phenomenon.

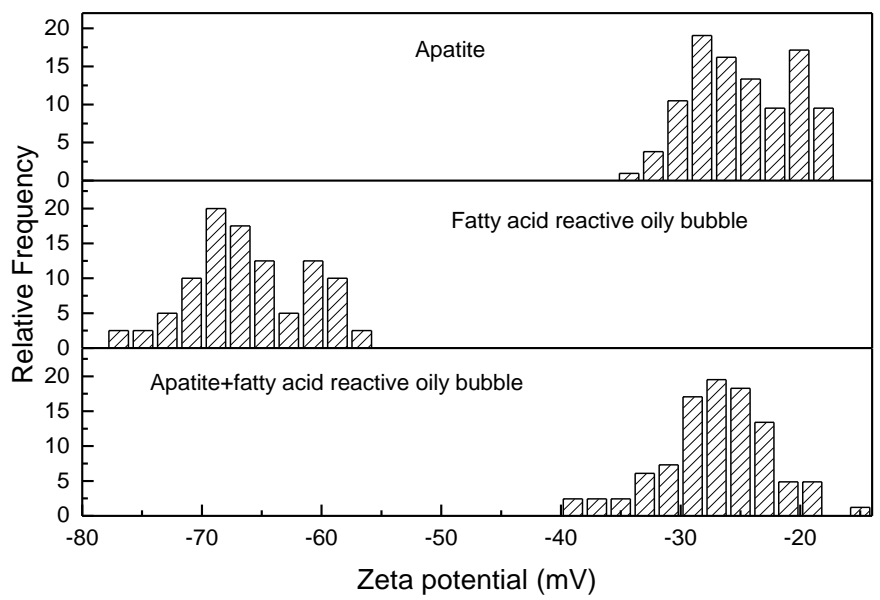

Fig.6 Zeta potential distribution of individual apatite and reactive oily bubble and their mixture at pH 9.01

Figure 6 shows that the zeta potential distribution of fatty acid reactive oily bubbles locates at around $-67 \mathrm{mV}$ and the apatite particles locates at around $-25 \mathrm{mV}$ at $\mathrm{pH}$ 9.01, respectively. An electrostatic repulsive force and thus an un-coagulation between the two surfaces are anticipated by the electrostatic interaction theory. However, the mixture of fatty acid reactive oily bubbles and apatite particles shows a single zeta potential distribution in Fig.6. A new surface with the similar or same electrical behavior is formed at $\mathrm{pH} 9.01$, which is the fatty acid reactive oily bubble coagulating with apatite particles. This result is opposite to the theoretical inference result, and yet is corresponding to the induction time result. The induction time and zeta potential distribution measurements shows the presence of a larger attraction force than the electrostatic repulsive force between apatite particles and fatty acid reactive oily bubbles.

\subsection{Micro flotation}

For a further investigation on the reactive oily bubbles collecting apatite particles, the micro flotation test was conducted with fatty acid reactive oily bubbles as carrier to 
floating apatite, and the results were compared to that of the air bubble floating apatite with fatty acid added in pulp shown in Fig. 7.

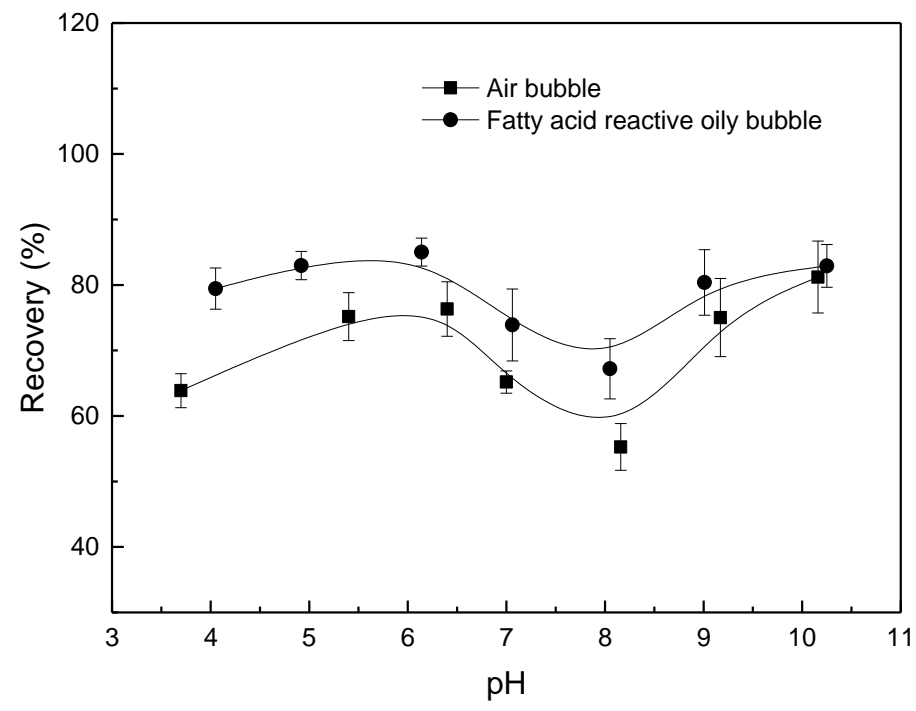

Fig.7 Flotation recovery of apatite using air bubble and fatty acid reactive oily bubble as a function of $\mathrm{pH}$

From Fig.7, whatever the $100 \mathrm{ppm}$ fatty acid is added in pulp or in the oil film of reactive oily bubble, the apatite can be floated among the $\mathrm{pH}$ range tested. However, the flotation recoveries of reactive oily bubbles are higher than the air bubbles, indicating a stronger collecting power of reactive oily bubbles than air bubbles. In a weak acid environment ( $\mathrm{pH} 5-6)$ and a weak alkaline environment ( $\mathrm{pH} 9-10)$, the flotation recovery of apatite from air bubbles or reactive oily bubbles can up to around $70 \%$, suggesting that the apatite is easy to floated in the two conditions. It may be inferred that reactive oily bubbles can affect the floating power rather than the flotation feasibility.

\subsection{Estimation of interaction energy}

\subsubsection{Electrostatic interaction between fatty acid reactive oily bubbles and apatite} particles

The surface charge of apatite is due to the generated hydroxylated surface by apatite reacting with water, which shows different charged surface in different $\mathrm{pH}$ solutions. From Fig.2, the zeta potential of fatty acid reactive oily bubble is $-13.56 \mathrm{mV}$ and $-69.77 \mathrm{mV}$, and the zeta potential of apatite particles is $6.38 \mathrm{mV}$ and $-26.97 \mathrm{mV}$ at $\mathrm{pH}$ 3.12 and 9.01, respectively. According to this results, the electrostatic interaction energy between fatty acid reactive oily bubbles and apatite particles was calculated by Eq.(2) as a function of separation distance shown in Fig. 10. 


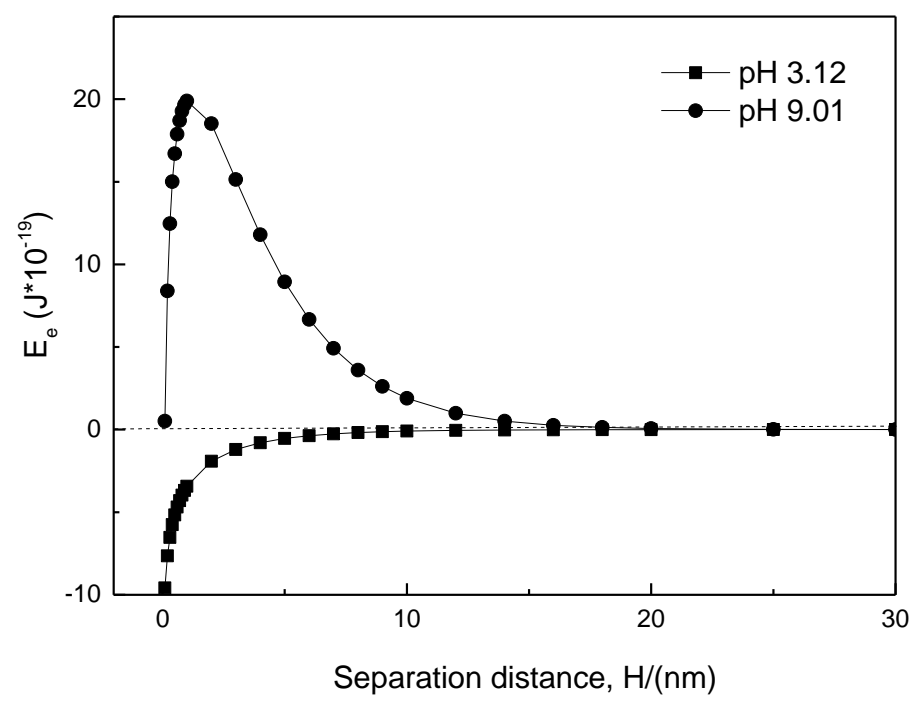

Fig.10 Electrostatic interaction between apatite and reactive oily bubble at $\mathrm{pH} 3.12$ and 9.01

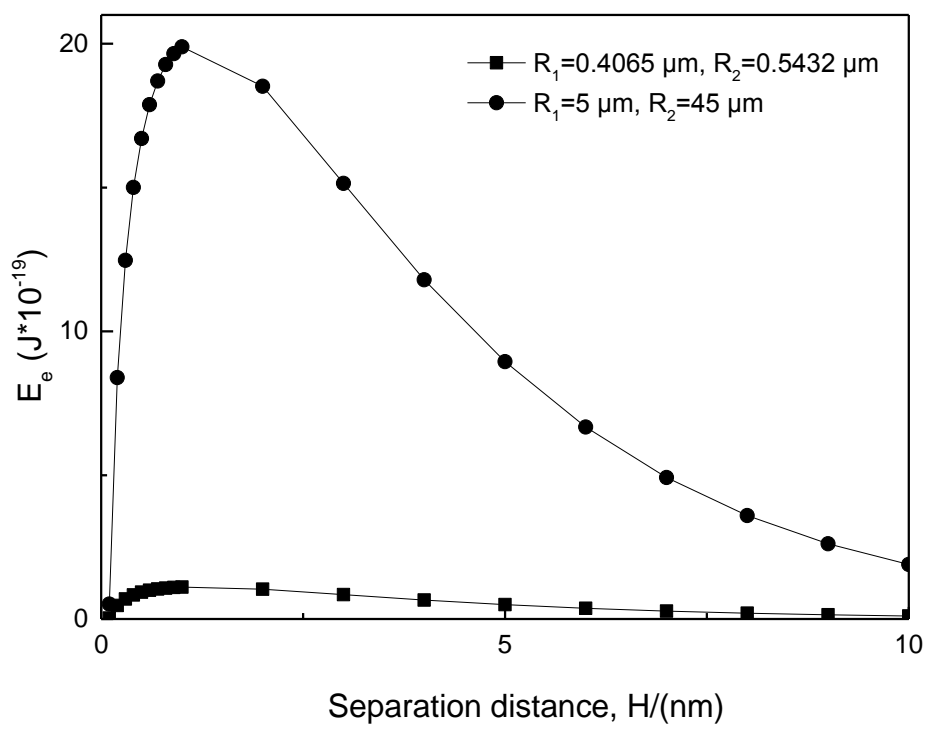

Fig.11 Electrostatic interaction between apatite and reactive oily bubble in different particle size conditions

At $\mathrm{pH}$ 3.12, an electrostatic attractive force is anticipated by the electronegativity surface of fatty acid reactive oily bubbles and the electropositivity surface of apatite particles from Fig.2, which is consistent with the electrostatic energy calculation in Fig.10. At pH 9.01, an electrostatic repulsive force is deduced by Fig.2 and calculated in Fig.10. Furthermore, the absolute force of electrostatic repulsive force at $\mathrm{pH} 9.01$ is larger than the electrostatic attractive force at $\mathrm{pH} 3.12$, resulting from that the absolute values of zeta potential of the two particles are higher in alkaline condition. 
Fig.11 shows the electrostatic interaction energy between different diameter fatty acid reactive oily bubbles and apatite particles at $\mathrm{pH} 9.01$ as a function of separation distance. A positive electrostatic interaction energy is calculated, suggesting an electrostatic repulsive force between reactive oily bubbles and apatite particles. With the increase of particle size, the electrostatic interaction energy keeps the same positive value, and its absolute value increases, indicating that the nature of the electrostatic interaction force, i.e., attractive force or repulsive force, cannot be altered by the particle size, but affected by the surface property.

\subsubsection{London-van der Waals interaction between fatty acid reactive oily bubbles and}

\section{apatite particles}

The London-van der Waals force between fatty acid reactive oily bubbles/air bubbles and apatite particles can be calculated by the Eq. (3). The Hamaker constants, $A_{132}$ for reactive oily bubbles system, and $A_{132}$ ' for air bubble system were calculated by Eq.(4) shown in Table 2.

Table 2 Hamaker constants $(A)$

\begin{tabular}{cc}
\hline Material & $A(\mathrm{~J})$ \\
\hline Water & $3.70 \times 10^{-20}$ \\
Apatite & $8.00 \times 10^{-20}$ \\
Air bubble & 0 \\
Fatty acid reactive oily bubble & $4.50 \times 10^{-20}$ \\
$A_{132}$ & $1.79 \times 10^{-21}$ \\
$A_{132}$, & $-1.74 \times 10^{-20}$ \\
\hline
\end{tabular}

The Hamaker constant of water is calculated by L. M. S. de Mesquita and his colleagues [38]. The Hamaker constant of reactive oily bubble is substituted by the octane's due to the similar chemical formula of fatty acid and octane [17]. The Hamaker constant of phosphate ore replaces the apatite's, $8.00 \times 10^{-20} \mathrm{~J}[39,40]$.

From the Hamaker constants calculation, $A_{132}$ is positive value in fatty acid reactive oily bubbles flotation system, and $A_{132}$ ' is negative value in air bubbles flotation system due to 0 value of air bubble's hamaker constant. According to Eq. (3), a negative London-van der Waals energy between the fatty acid reactive oily bubble and the apatite particle is calculated suggesting an attractive force, but oppositely, there is a repulsive force between the air bubble and the apatite particle. It can be seen that the oil film containing fatty acid covered on reactive oily bubbles changes the 
surface property of air bubbles and so as to change the London-van der Waals interaction force from repulsive to attractive, which further confirms a stronger collecting power of fatty acid reactive oily bubbles than air bubbles to apatite particles.

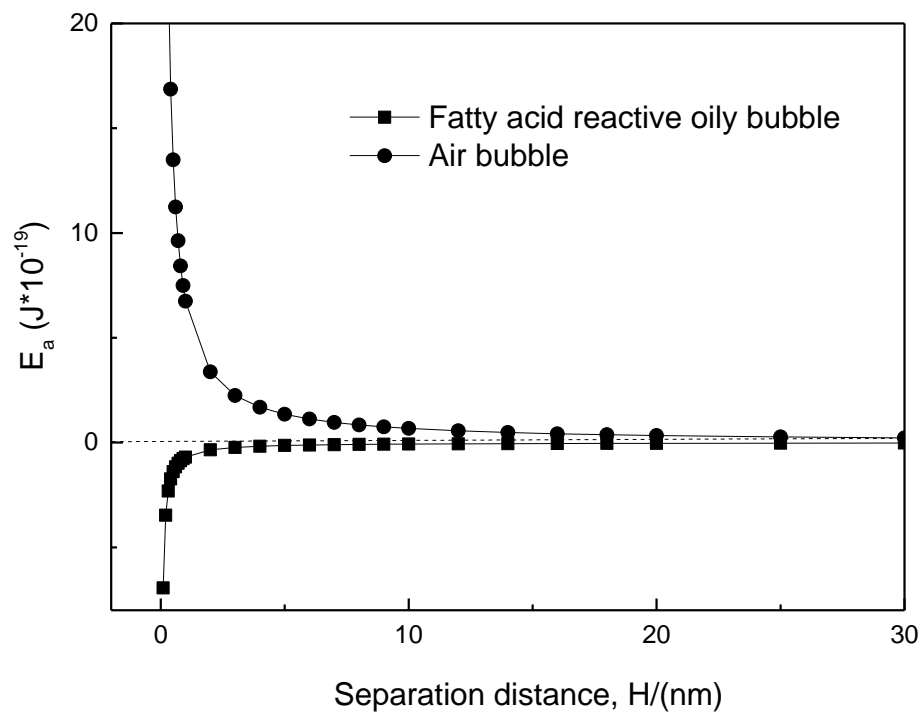

Fig.8 Van der Waals interaction between apatite and different bubbles $\left(R_{1}=0.4065 \mu \mathrm{m}\right.$ and

$$
R_{2}=0.5432 \mu \mathrm{m} \text { ) }
$$

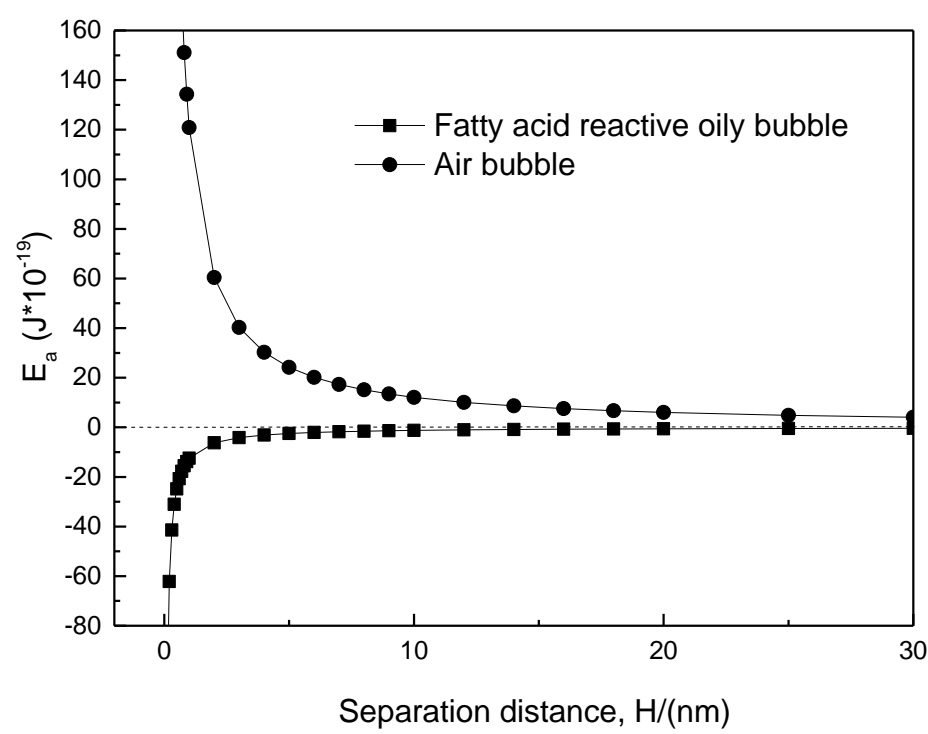

Fig.9 Van der Waals interaction between apatite and different bubbles $\left(R_{1}=5 \mu \mathrm{m}\right.$ and $\left.R_{2}=45 \mu \mathrm{m}\right)$

The particle size of fatty acid reactive oily bubbles and apatite particles in the Zeta potential test are $0.4065 \mu \mathrm{m}\left(R_{1}\right)$ and $0.5432 \mu \mathrm{m}\left(R_{2}\right)$, respectively. The calculation 
results of Van der Waals interaction energy is shown in Fig. 8. For micro flotation, the diameter of bubbles and apatite particles is selected as $R_{1}=5 \mu \mathrm{m}$ and $R_{2}=45 \mu \mathrm{m}$, respectively, where the calculation results as a function of separation distance are shown in Fig.9.

As shown in Fig.8 and Fig.9, no matter what the particle size is, it is Van der Waals attractive force between the fatty acid reactive oily bubble and the apatite particle, and is Van der Waals repulsive force between the air bubble and the apatite particle, which is consistent with the deduced results by Hamaker constant. Both the Van der Waals attractive force and repulsive force increase with the increase of particle size in the same separation distance. Compared to the air bubble, the surface property of fatty acid reactive oily bubble as carrier is changed by the oil film containing fatty acid in flotation. The Van der Waals repulsive force of carrier (air bubbles) to apatite particles is transformed to Van der Waals attractive force (fatty acid reactive oily bubbles), which enhance the collecting power of the bubble carrier to mineral particles and is benefit to the flotation.

\subsubsection{Hydrophobic interaction between fatty acid reactive oily bubbles and} apatite particles

The hydrophobic interaction energy between fatty acid reactive oily bubbles and apatite particles as a function separation distance is calculated by Eq. (8) and the results are shown in Fig.12. $K_{132}$ is calculated by E.q(9) and shown in Table 3.

Table 3 Calculation of $K_{132}$

\begin{tabular}{cccc}
\hline $\mathrm{pH}$ & $\begin{array}{c}K_{131^{\mathrm{a}}} \\
\left(\times 10^{17} \mathrm{~J}\right)\end{array}$ & $\begin{array}{c}K_{232}{ }^{\mathrm{b}} \\
\left(\times 10^{21} \mathrm{~J}\right)\end{array}$ & $\begin{array}{c}K_{132} \\
\left(\times 10^{19} \mathrm{~J}\right)\end{array}$ \\
\hline 3.12 & 0.4 & 1.45 & 0.76 \\
9.01 & 0.4 & 22.5 & 3.00 \\
\hline
\end{tabular}

${ }^{\mathrm{a}}$ from Aksoy [35].

${ }^{\mathrm{b}}$ from Yoon and Luttrell [41]. 


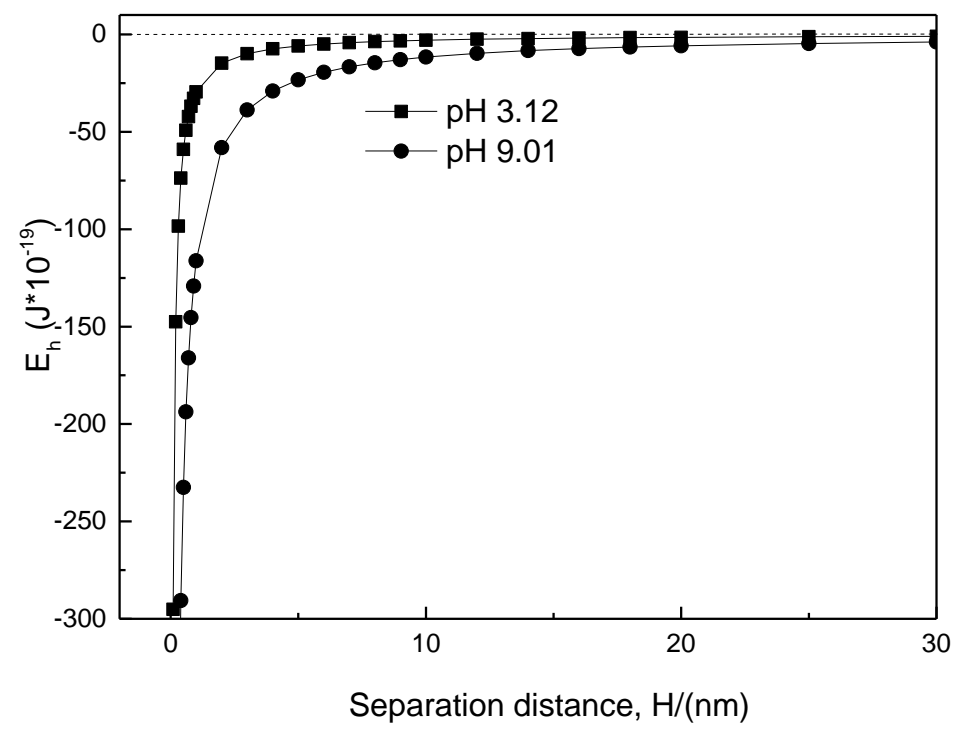

Fig.12 Hydrophobic interaction between apatite and reactive oily bubble at $\mathrm{pH} 3.12$ and 9.01

From Fig.12, the negative values of the hydrophobic interaction energy among the tested separation distance indicate a hydrophobic attractive force between fatty acid reactive oily bubbles and apatite particles at $\mathrm{pH} 3.12$ and 9.01. Comparing Fig.12 to Figs. 10 and 11, it is found that the absolute value of the hydrophobic energy is larger than the electrostatic energy. A smaller hydrophobic interaction energy is calculated by the smaller $K_{232}$ at $\mathrm{pH} 3.12$ than at $\mathrm{pH}$ 9.01. From Eq.(3) and Eq.(8), the hydrophobic energy can be compared to the electrostatic energy through comparing the hydrophobic constant $\left(K_{132}\right)$ to the hamaker constant $\left(A_{132}\right)$. The $K_{132}$ of fatty acid reactive oily bubbles and apatite particles is $10^{-19} \mathrm{~J}$ lower than the $A_{132}, 10^{-21} \mathrm{~J}$, and thus a larger hydrophobic interaction force than the electrostatic interaction force can be directly deduced. The results suggest that the hydrophobic interaction plays an more important role in the attachment of fatty acid reactive oily bubbles to the apatite surface than the electrostatic interaction. 


\subsubsection{Interaction energy between fatty acid reactive oily bubbles and apatite particles}

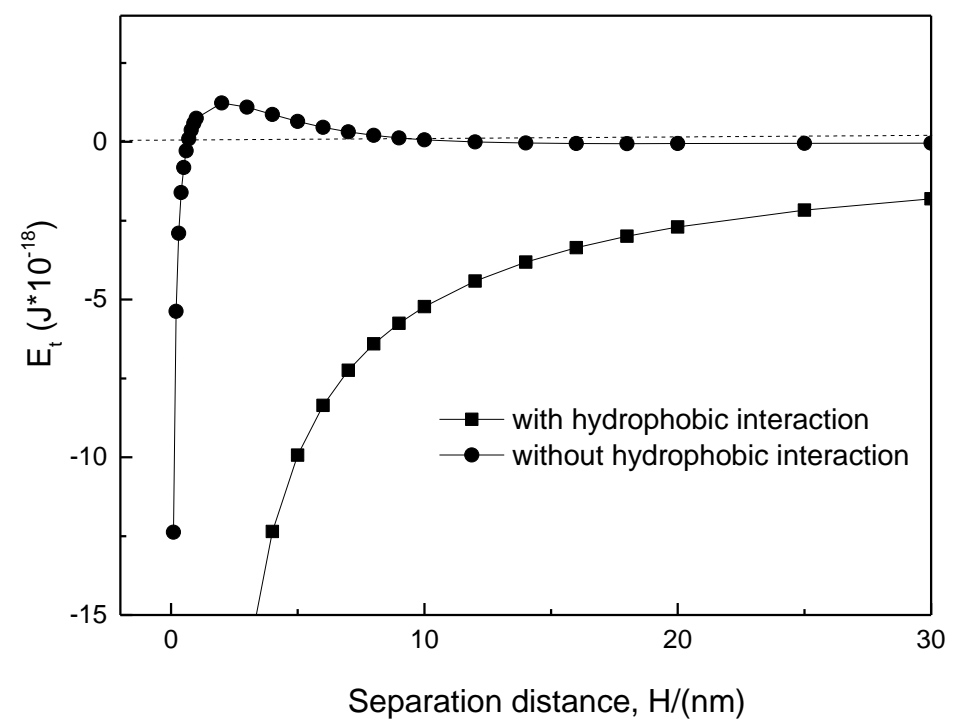

Fig.13 Interaction between apatite and reactive oily bubble at $\mathrm{pH} 9.01$

The total interaction energy between fatty acid reactive oily bubbles and apatite particles at $\mathrm{pH} 9.01$ as a function of separation distance is calculated with and without the hydrophobic interaction energy shown in Fig.13 together. Without the hydrophobic interaction energy consideration, the total interaction energy increases firstly with the increase of separation distance from negative value to positive value, and then decreases to negative value again. For $0.7-10 \mathrm{~nm}$, the fatty acid reactive oily bubble is repulsive to the apatite particle at $\mathrm{pH} 9.01$, which is opposite to the induction time and zeta potential distribution results in Figs. 4 and 6. The DLVO theory is fail to illustrate the coagulation phenomena observed in the induction time and zeta potential distribution measurements. With the hydrophobic interaction energy consideration, a negative total interaction energy indicates an attractive force and further a coagulation between fatty acid reactive oily bubbles and apatite particles at $\mathrm{pH}$ 9.01, which is consistent with the induction time and zeta potential distribution results in Figs. 4 ad 6. This result demonstrates the importance of hydrophobic interaction energy in the fatty acid reactive oily bubble system.

\section{Conclusions}

It was shown in this study that the surface property of reactive oily bubble can be flexibly controlled by altering the collector and its concentration in the oil film 
covered on reactive oily bubble surface. A strong interaction between fatty acid reactive oily bubbles and apatite particles among $\mathrm{pH}$ 4.0-11.2 was observed by induction time and zeta potential distribution experiments, which commendably explains the better flotation performance of reactive oily bubble than air bubble. However, an un-coagulation between reactive oily bubbles and apatite particles is deduced by the DLVO theory at $\mathrm{pH} 9.01$, suggests the failing of DLVO theory in the reactive oily bubble system among the alkaine $\mathrm{pH}$ range. A non-DLVO force, the hydrophobic interaction, is adopted to illustrate the coagulation phenomena at $\mathrm{pH}$ 9.01. The results showed the extended DLVO theory is suitable for the fatty acid reactive oily bubble-apatite flotation system, and the superiority of fatty acid reactive oily bubble system was confirmed again.

\section{Acknowledgement}

Financial supports for this work from National Science Foundation of China (51404171) (China), National Science Foundation of Hubei (2015CFB662) (China), Hubei Provincial Department of Education (T201506) (China) and Wuhan Institute of Technology (237850) (China) are greatly appreciated.

\section{References}

[1] F. Zhou, L. Wang, Z. Xu, Q. Liu, R. Chi, Reactive oily bubble technology for flotation of apatite, dolomite and quartz. Int. J. Miner. Process. 134(2015) 74-81.

[2] J. Liu, T. Mak, Z. Zhou, Z. Xu, Fundamental study of reactive oily-bubble flotation. Miner. Eng. 15.9(2002)667-676.

[3] T. K. Mitchell, A. V. Nguyen, G. M. Evans, Heterocoagulation of chalcopyrite and pyrite minerals in flotation separation. Adv. Colloid Interface Sci. 114-115 (2005) 227-237.

[4] R. C. Santana, C. R. Duarte, C. H. Ataide, M. A. S. Barrozo, Flotation selectivity of phosphate ore: Effects of particle size and reagent concentration. Sep. Sci. Technol. 46.9(2011) 1511-1518.

[5] F. Zhou, L. Wang, Z. Xu, Q. Liu, M. Deng, R. Chi, Application of reactive oily bubble to bastnaesite flotation. Miner. Eng. 64.9(2014) 139-145.

[6] J. Liu, Z. Xu, J. Masliyah, Interaction between bitumen and fines in oil sands extraction system: implication to bitumen recovery. Can. J. Chem. Eng. 82.4(2008)655-666.

[7] Z. Xu, J. Liu, J. Choung, Z. Zhou, Electrokinetic study of clay interactions with coal in flotation. Int. J. Miner. Process. 68(2003) 183-196. 
[8] R. Yoon, J. L. Yordan, Induction time measurements for the quartz-amine flotation system. J. Colloid Interf. Sci. 141.2(1991)374-383.

[9] Y. Ye, S. M. Khandrika, J. D. Miller, Induction-time measurements at a particle bed. Int. J. Miner. Process. 25.3-4(1989)221-240.

[10] P.T.L. Koh, L.K. Smith, The effect of stirring speed and induction time on flotation. Miner. Eng. 24.5 (2011) 442-448.

[11] M. Bendersky, J. M. Davis, DLVO interaction of colloidal particles with topographically and chemically heterogeneous surfaces. J. Colloid Interf. Sci. 353.1 (2011) 87-97.

[12] J. Wang, R.H. Yoon, J. Morris, AFM surface force measurements conducted between gold surfaces treated in xanthate solutions. Int. J. Miner. Process. 122(2013)13-21.

[13] M. Siebentritt, P. Volovitch, K. Ogle, G. Lefevre, Surface potential of hematite particles in high concentration electrolytes: Electroacoustic measurements and suspension stability. Colloid Surface A. 443(2014)338-344.

[14] M. Zhang, Q. Liu, J. Liu, Extended DLVO theory applied to coal slime-water suspensions. J. Cent. South Univ. 19.12(2012)3558-3563.

[15] G. Hwang, I. Ahn, B. J. Mhin, J. Kim, Adhesion of nano-sized particles to the surface of bacteria: Mechanistic study with the extended DLVO theory. Colloid Surface B. 97(2012)138-144.

[16] Y. Li, Application of electroacoustic effects in the determination of particle properties. Chemistry. 64(2001)1(in China).

[17] N. A. Mishchuk, The model of hydrophobic attraction in the framework of classical DLVO forces. Adv. Colloid Interface Sci. 168.1-2(2011)149-166.

[18] H. Schubert, On the optimization of hydrodynamics in fine particle flotation. Miner. Eng. 21.12-14(2008)930-936.

[19] K. Darcovich, A hydrodynamic simulation of mineral flotation. Part I: the numerical model. Powder Technol. 83(1995)211-224.

[20] K. Darcovich, C. E. Capes, A hydrodynamic simulation of mineral flotation. Part II: surface chemical effects for coal-oil agglomerate flotation. Powder Technol. 83(1995)225-232.

[21] G. S. Dobby, J. A. Finch, Particle size dependence in flotation derived from a fundamental model of the capture process. Int. J. Miner. Process. 21.3-4(1987) 241-260.

[22] Z. Xu, R. H. Yoon, The role of hydrophobia interactions in coagulation. J. Colloid Interf. Sci.132.2(1989)532-541.

[23] H. Yotsumoto, R. H. Yoon, Application of extended DLVO theory: I. Stability of rutile suspensions. J. Colloid Interf. Sci. 157.2(1993)426-433. 
[24] H. Yotsumoto, R. H. Yoon, Application of extended DLVO theory: II. Stability of silica suspensions. Journal of colloid and interface science, J. Colloid Interf. Sci. 157.2(1993)434-441.

[25] J. Israelachvili, R. Pashley, The hydrophobic interaction is long range, decaying exponentially with distance. Nature. 300(1982)341-342.

[26] R. M. Pashley, P. M. McGuiggan, B. W. Ninham, D. F. Evans, Attractive forces between uncharged hydrophobic surfaces: direct measurements in aqueous solution. Science. 229.4718(1985) 1088-1089.

[27] P. M. Claesson, P. C. Herder, C. E. Blom, B. W. Ninham, Interactions between a positively charged hydrophobic surface and a negatively charged bare mica surface. J. Colloid Interf. Sci.118(1)(1986)68-79.

[28] Y. H. Tsao, S. X. Yang, D. F. Evans, Interactions between hrdrophobic surface. Dependence on temperature and alkyl chain length. Langmuir. 7.12(1991)3154-3159.

[29] R. H. Yoon, S. A. Ravishankar, Long-range hrdrophobic force between mica surfaces in dodecylammonium chlorides solutions in the presence of dodecanol. J. Colloid Interf. Sci. 179.2(1996)391-402.

[30] P. M. Claesson, H. K. Christenson, Very long range attractive forces between uncharged hydrocarbon and fluorocarbon surfaces in water. J. Phys. Chem. 92.6(1988)1650-1655.

[31] R. Varbanov, E. Forssberg, M. Hallin, On the modelling of the flotation process. Int. J. Miner. Process. 37.1-2(1993)27-43.

[32] F. G. Paulsen, R. Pan, D. W. Bousfield, E. V. Thompson, The Dynamics of bubble/particle attachment and the application of two disjoining film rupture models to flotation.: I. Nondraining Model. Journal of Colloid and Interface Science, J. Colloid Interf. Sci. 178.(2(1996) 400-410.

[33] R. H. Yoon, L. Mao, Application of extended DLVO theory, IV: Derivation of flotation rate equation from first principles. J. Colloid Interf. Sci. 181.2(1996) 613-626.

[34] L. Mao, Application of Extended DLVO Theory: Modeling of Flotation and Hydrophobicity of Dodecane, Canada: University of Alberta, 1998(Doctoral dissertation).

[35] B. S. Aksoy, Hydrophobic forces in free thin films of water in the presence and absence of surfactants, America: Virginia Poly technic Institute and State University, 1997 (Doctoral dissertation).

[36] J. Shibata, D.W. Fuerstenau, Flocculation and flotation characteristics of fine hematite with sodium oleate. Int. J. Miner. Process. 72(2003)25-32. 
[37] C. P. Brennand, j. k. Ha, R. C. Lindsay, Aroma properties and thresholds of some branched-chain and other minor volatile fatty acid occurring in milkfat and meat lipids. J. Sens. Stud. 4.2(1989)105-120.

[38] L. M. S. Mesquita, F. F. Lins, M. L. Torem, Interaction of a hydrophobic bacterium strain in a hematite-quartz flotation system. Int. J. Miner. Process. 71.1-4(2003)71:31.

[39] C. R. N. Silva, V. S. Negrini, M. L. Aguiar, J. R. Coury, Influence of gas velocity on cake formation and detachment. Powder Technol. 101.2(1999)165-172.

[40] M. A. Felicetti, F. Piantino, J. R. Coury, Influence of removal time and particle size on the particle substrate adhesion force. Braz. J. Chem. Eng. 25.1(2008) 71-82.

[41] R. H. Yoon, G. H. Luttrell, Development of the Selective Hydrophobic Coagulation Process. Report No. DOE/PC/91164-T1, Virginia Center for Coal and Minerals Processing, Blacksburg, VA (United States), 1992. 

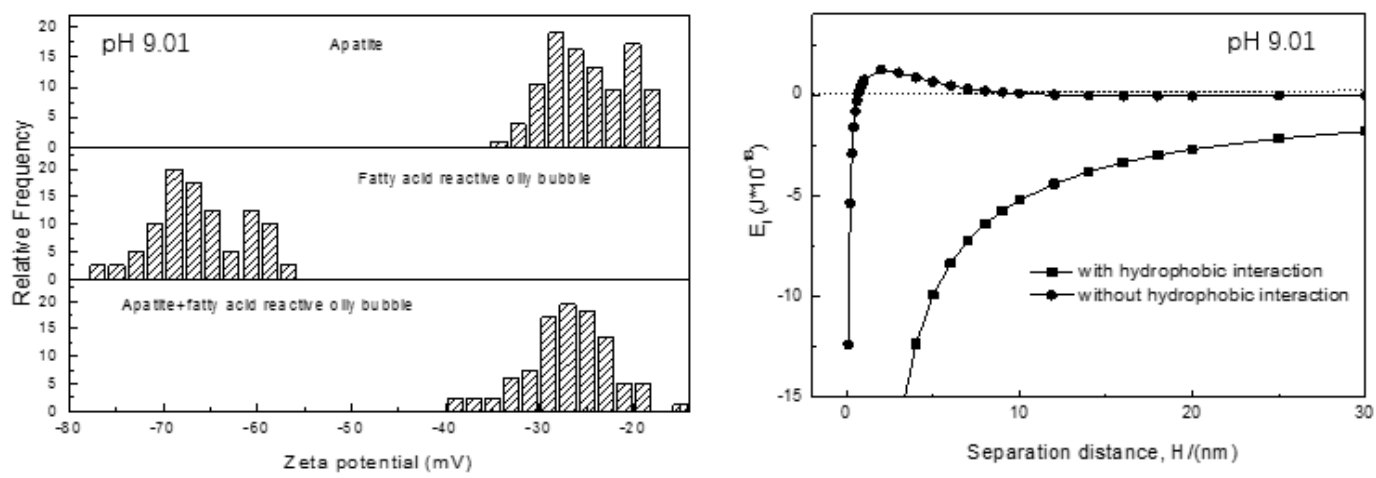